\title{
COMMENTARY
}

\section{Protease-activated receptor-1: key player in the sepsis coagulation-inflammation crosstalk}

\author{
Karim Asehnoune ${ }^{* 1}$ and Pierre Moine ${ }^{2}$ \\ See related research by Schouten et al., http://ccforum.com/content/16/6/R238
}

\begin{abstract}
Protease-activated receptors (PARs) belong to the family of $\mathrm{G}$ protein-coupled receptors. Among the four members, PAR1 plays a major role in orchestrating the interactions between coagulation and inflammation. PAR1 has opposing functions during sepsis, and PAR1 blockade or activation may be alternatively beneficial at early or late stages of different sepsis models. Studying molecular mechanisms of the crosstalk between inflammation and coagulation may lead to the identification of new targets for therapies in sepsis. However, the time-dependent switch of PAR1 from an exacerbating proinflammatory receptor to a protective anti-inflammatory receptor needs to be investigated before clinical trials can be recommended. Finally, as PAR1 seems to play a singular role in Streptococcus pneumoniae-induced sepsis through a crosstalk between PAR1 and platelet-activating factor receptor, the exact role of PAR1 needs to be investigated in other models of sepsis.
\end{abstract}

\section{Introduction}

In the previous issue of Critical Care, Schouten and colleagues show the critical involvement of proteaseactivated receptor (PAR)-1 in a lethal Streptococcus pneumoniae pneumonia model [1]. PARs belong to the family of $\mathrm{G}$ protein-coupled receptors [2]. Four PARs are currently known (PAR1, PAR2, PAR3, PAR4). In contrast with other G protein-coupled receptors, PARs are not activated in vivo by binding of a soluble ligand but, instead, are activated by proteolysis triggered by extracellular proteases. PARs use a fascinating mechanism to convert an extracellular proteolytic cleavage event into a

*Correspondence: karim.asehnoune@gmail.com

'Service d'Anesthésie réanimation, CHU de Nantes, 1 place Alexis Ricordeau, 44093 Nantes Cedex 1, France

Full list of author information is available at the end of the article transmembrane/intracellular signal: the receptors carry their own tethered ligands, which remain cryptic until unmasked by receptor $\mathrm{N}$-terminal cleavage, and then an intramolecular rearrangement allows the ligand and the receptor moieties to interact. A considerable body of evidence supports a prominent role for PARs in a variety of human physiological and pathophysiological processes, and thus substantial attention has been paid to develop new drug-like molecules either activating or blocking PARs [2].

\section{PAR1 is involved in the interactions between inflammation and coagulation}

The crosstalk of inflammation and coagulation has emerged as a major mechanism controlling the host response to invading microorganisms, and poor regulation of this mechanism is held responsible for the occurrence of multiple organ failure and eventually death in patients with severe sepsis/septic shock. PAR1 plays a major role in orchestrating the interplay between coagulation and inflammation [2-5].

PAR1 is the primary cell-surface receptor responsible for thrombin-mediated platelet aggregation in humans, but is also activated by many other proteases - including activated protein $\mathrm{C}$ (APC) and its receptor, the endothelial protein $C$ receptor. All of these different proteases, which are released during activation of the clotting cascade, can regulate PAR signalling by either activation or inactivation. PAR1 then has an intriguing dual function: PAR1 activation by thrombin results in a proinflammatory/endothelial-permeability enhancing response, while the same receptor activated by APC/endothelial protein $\mathrm{C}$ receptor results in an anti-inflammatory, endothelial-integrity preserving response [3]. Nevertheless, understanding the role of PAR1 signalling in sepsis remains complex due to the multiple and, in part, opposite effects ascribed to this receptor.

A further complexity is present when many types of cells, all present in the same environment, express this receptor - platelets, leukocytes, macrophages, endothelial cells, epithelial cells and fibroblasts, for example. Moreover, thrombin has a much higher affinity for PAR1 
with a higher catalytic efficiency relative to APC. How APC activates PAR1 is unclear when thrombin is also present in the same environment.

Finally, PAR1 can take up multiple conformational states, each state triggering different downstream signalling pathways and cellular responses. For example, compartmentalisation of PAR1 in lipid rafts and localisation to caveolae are critical for the APC-biased agonist of PAR1 [2].

\section{PAR1 and Streptococcus pneumoniae pneumonia}

In the lungs, the role of PARs in inflammatory processes remains controversial [4]. PAR1 activation contributes to the pathogenesis of influenza A virus infection [6]. PAR1 signalling inhibition decreases inflammation, early virus replication and mortality after infection with multiple influenza A virus strains, and is effective even when dosing was initiated at day 3 after inoculation. Additionally, PAR1 activation exacerbates ventilation injuryinduced pulmonary oedema [7]. Also, $\mathrm{Parl}^{-/-}$mice are protected from ventilation lung injury in a setting of high-tidal volume ventilation and bleomycin-induced lung injury [7-9]. These observations suggest that PAR1 signalling contributes to proinflammatory responses to injury in the lungs.

In the study by Schouten and colleagues, PAR1 impairs the host defence response, as reflected by a reduced lethality, lower bacterial loads, less pulmonary neutrophil influx and less lung damage in PAR1 knockout mice [1]. Even if the mechanisms underlying these differences remain to be elucidated, crosstalk between PAR1 and platelet-activating factor receptor (PAFR) may partly explain these differences. PAR1 and PAFR have been shown to cooperate together, as PAR1 induced the expression of platelet-activating factor and PAFR [10]. Indeed, PAFR plays a crucial role in the pathogenesis of pneumococcal disease. The biological activity of plateletactivating factor is mainly determined by phosphorylcholine, which binds specifically to PAFR. Phosphorylcholine is also a prominent part of the cell wall of S. pneumoniae and specifically binds to PAFR expressed on human respiratory epithelial cells, which facilitates pneumococcal entry into these cells and transcytosis to the basal surface of endothelial cells [11]. Using PAFR knockout mice, the same group demonstrated that PAFR was used by $S$. pneumoniae to induce severe lethal pneumonia, as reflected by a reduced mortality, attenuated bacterial outgrowth in the lungs and diminished dissemination of the infection in PAFR knockout mice [11]. PAR1 may then amplify PAFR-dependent pneumococcal dissemination and induce severe pneumonia. Further studies are definitely needed to dissect the exact PAR1 signalling mechanisms in S. pneumoniae pneumonia. Nevertheless, as underlined by the authors, the therapeutic effect and impact of PAR1 signalling inhibition with concurrent antibiotic treatment for S. pneumoniae pneumonia needs to be evaluated.

Other studies examining the role of PAR1 in sepsis have revealed conflicting results. Whereas studies using blocking agents confirmed the present results, others showed detrimental effects. These discrepancies may be explained by the different sepsis models (pneumonia, polymicrobial sepsis induced by caecal ligation and puncture and endotoxinemia), different severity and inoculum (lethal dose 90 vs. lethal dose 100) and different stages of the disease. PAR1 has opposing, temporally controlled functions during the progression of the sepsis, and PAR1 blockade or activation may be alternatively beneficial at early or late stages of different sepsis models. The complexity of PAR1 signalling mechanisms supports the assessment of the immunological status of the patients. Immunological monitoring could help to guide therapies with immunomodulatory drugs with an antiinflammatory effect or an immunostimulatory effect [12].

\section{Conclusion}

Increased insight into the molecular mechanisms of the tight relationship between inflammation and coagulation may lead to the identification of new targets for therapies that can modify the excessive activation that leads to dysregulation of these systems. PAR1 has a pivotal role in mediating protective APC effects. The time-dependent switch of PAR1 from an exacerbating proinflammatory receptor to a protective anti-inflammatory receptor needs to be investigated in other experimental models before clinical trials can be recommended.

\section{Abbreviations}

APC, activated protein C; PAFR, platelet-activating factor receptor; PAR, protease-activated receptor.

Competing interests

The authors declare that they have no competing interests.

\section{Author details}

'Service d'Anesthésie réanimation, CHU de Nantes, 1 place Alexis Ricordeau, 44093 Nantes Cedex 1, France. ${ }^{2}$ Department of Anesthesiology, School of Medicine, University of Colorado Denver, Mail Stop B113, 12401 East $17^{\text {th }}$ Avenue, Aurora, CO 80045, USA

Published: 26 February 2013

\section{References}

1. Schouten M, van't Veer C, Roelofs JJ, Levi M, van der Poll T: Proteaseactivated receptor- 1 impairs host defense in murine pneumococcal pneumonia: a controlled laboratory study. Crit Care 2012, 16:R238.

2. Adams MN, Ramachandran R, Yau M-K, Suen JY, Fairlie DP, Hollenberg MD, Hooper JD: Structure, function and pathophysiology of protease activated receptors. Pharmacol Ther 2011, 130:248-282

3. Petäjä J: Inflammation and coagulation. An overview. Thromb Res 2011, 127(Suppl 2):S34-S37.

4. Peters T, Henry PJ: Protease-activated receptors and prostaglandins in inflammatory lung disease. Br J Pharmacol 2009, 158:1017-1033.

5. van der Poll T, Levi M: Crosstalk between inflammation and coagulation: the lessons of sepsis. Curr Vasc Pharmacol 2012, 10:632-638. 
6. Khoufache K, Berri F, Nacken W, Vogel AB, Delenne M, Camerer E, Coughlin SR, Carmeliet P, Lina B, Rimmelzwaan GF, Planz O, Ludwig S, Riteau B: PAR1 contributes to influenza A virus pathogenicity in mice. J Clin Invest 2013, 123:206-214.

7. Jenkins RG, Su X, Su G, Scotton CJ, Camerer E, Laurent GJ, Davis GE, Chambers RC, Matthay MA, Sheppard D: Ligation of protease-activated receptor 1 enhances alpha(v)beta6 integrin-dependent TGF-beta activation and promotes acute lung injury. $J$ Clin Invest 2006, 116:1606-1614.

8. Mercer PF, Deng X, Chambers RC: Signaling pathways involved in proteinase-activated receptor 1 -induced proinflammatory and profibrotic mediator release following lung injury. Ann N Y Acad Sci 2007, 1096:86-88.

9. Chen D, Carpenter A, Abrahams J, Chambers RC, Lechler Rl, McVey JH, Dorling A: Protease-activated receptor 1 activation is necessary for monocyte chemoattractant protein 1-dependent leukocyte recruitment in vivo. J Exp Med 2008, 205:1739-1746.

10. Melnikova VO, Balasubramanian K, Villares GJ, Dobroff AS, Zigler M, Wang H, Petersson F, Price JE, Schroit A, Prieto VG, Hung M-C, Bar-Eli M: Crosstalk between protease-activated receptor 1 and platelet-activating factor receptor regulates melanoma cell adhesion molecule (MCAM/MUC18) expression and melanoma metastasis. J Biol Chem 2009, 284:28845-28855.

11. Rijneveld AW, Weijer S, Florquin S, Speelman P, Shimizu T, Ishii S, van der Poll T: Improved host defense against pneumococcal pneumonia in plateletactivating factor receptor-deficient mice. J Infect Dis 2004, 189:711-716.

12. Almansa R, Wain J, Tamayo E, Andaluz-Ojeda D, Martin-Loeches I, Ramirez P, Bermejo-Martin JF: Immunological monitoring to prevent and treat sepsis. Crit Care 2013, 17:109.

doi:10.1186/cc12502

Cite this article as: Asehnoune K, Moine P: Protease-activated receptor-1: key player in the sepsis coagulation-inflammation crosstalk. Critical Care 2013, 17:119. 